\title{
IRREDUCIBLE COMPLEXITY OF ITERATED SYMMETRIC BIMODAL MAPS
}

\author{
J. P. LAMPREIA, R. SEVERINO, AND J. SOUSA RAMOS
}

Received 9 March 2004

We introduce a tree structure for the iterates of symmetric bimodal maps and identify a subset which we prove to be isomorphic to the family of unimodal maps. This subset is used as a second factor for a $*$-product that we define in the space of bimodal kneading sequences. Finally, we give some properties for this product and study the $*$-product induced on the associated Markov shifts.

\section{Introduction and preliminary definitions}

The concept of irreducible complexity of a biological system was introduced by Behe [1] in 1996. His point of view is that an organism consisting of a finite, possibly very large, number of independent components, coupled together in some way, exhibits irreducible complexity if, by removing any of its components, the reduced system no longer functions meaningfully. Using the language of nonlinear dynamics and chaos theory, Boyarsky and Góra [2] reinterpreted Behe's definition from a Markov transition matrix perspective by saying that a system is irreducibly complex if the associated transition matrix is primitive but no principal submatrix is primitive.

It is our conviction that the concept of reducible complexity of a dynamical system can also be interpreted in terms of a factorization: within Milnor and Thurston's kneading theory framework and the topological classification obtained from it, Derrida et al. [4] introduced a $*$-product between unimodal kneading sequences for which it was possible to prove that the topological entropy, a measure of complexity, of a factorizable system is equal to the topological entropy of one of the factors. Despite of a larger number of its components, the complexity of the system remains the same whenever its irreducible component, a factor of the product, does not change.

Some years later, Lampreia et al. [5] introduced a Markov transition matrix formalism associated with the kneading theory and a product between unimodal matrices corresponding to the Derrida-Gervois-Pomeau $*$-product. Then they proved that irreducible unimodal kneading sequences correspond to primitive Markov transition matrices. 
With this work we would like to introduce the generalization, for bimodal symmetric maps of the interval, of the $*$-product and the corresponding product between transition matrices.

Consider a two-parameter family $f_{a, b}$ of maps from the closed interval $I=\left[c_{0}, c_{3}\right]$ into itself, with two critical points, usually called the bimodal family of maps of the interval, see $[3,7,10]$. Once the parameters $(a, b)$ are fixed, the map $f_{a, b}$ is piecewise monotone, and hence $I$ can be subdivided in the following three subintervals: $L=\left[c_{0}, c_{1}\right]$, $M=\left[c_{1}, c_{2}\right]$, and $R=\left[c_{2}, c_{3}\right]$, where $c_{i}$ are the critical points or the extremal points, in such a way that the restriction of $f$ to each interval is strictly monotone. We will choose the family of maps such that the restrictions $f_{a, b \mid L}$ and $f_{a, b \mid R}$ are increasing and the restriction $f_{a, b \mid M}$ is decreasing.

For each value $(a, b)$, we define the orbits of the critical points by

$$
O\left(c_{i}\right)=\left\{x_{j}: x_{j}=f^{j}\left(c_{i}\right), j \in \mathbb{N}\right\}
$$

with $i=1,2$.

With the aim of studying the topological properties of these orbits we associate to each orbit $O\left(c_{i}\right)$ a sequence of symbols $S=S_{1} S_{2} \ldots S_{j} \ldots$, where $S_{j}=L$ if $f_{a, b}^{j}\left(c_{i}\right)<c_{1}, S_{j}=A$ if $f_{a, b}^{j}\left(c_{i}\right)=c_{1}, S_{j}=M$ if $c_{1}<f_{a, b}^{j}\left(c_{i}\right)<c_{2}, S_{j}=B$ if $f_{a, b}^{j}\left(c_{i}\right)=c_{2}$, and $S_{j}=R$ if $f_{a, b}^{j}\left(c_{i}\right)>c_{2}$. If we denote by $n_{M}$ the frequency of the symbol $M$ in a finite subsequence of $S$, we can define the $M$-parity of this subsequence according to whether $n_{M}$ is even or odd. In what follows (see [10]), we define an order relation in $\Sigma_{5}=\{L, A, M, B, R\}^{\mathbb{N}}$ that depends on the $M$-parity.

Let $V$ be a vector space of dimension three defined over the rationals having as a basis the formal symbols $\{L, M, R\}$; then to each sequence of symbols $S=S_{1} S_{2} \ldots S_{j} \ldots$ we can associate a sequence $\theta=\theta_{0} \ldots \theta_{j} \ldots$ of vectors from $V$, setting $\theta_{j}=\prod_{i=0}^{j-1} \epsilon\left(S_{i}\right) S_{j}$ with $j>0$, $\theta_{0}=S_{0}$ when $i=0$, and $\epsilon(L)=-\epsilon(M)=\epsilon(R)=1$, where to the symbols corresponding to the critical points $c_{1}$ and $c_{2}$ we associate the vectors $(L+M) / 2$ and $(M+R) / 2$. Thus $\epsilon(A)=\epsilon(B)=0$. Choosing then a linear order in the vector space $V$ in such a way that the base vectors satisfy $L<M<R$, we are able to order the sequence $\theta$ lexicographically, that is, $\theta<\bar{\theta}$ if and only if $\theta_{0}=\bar{\theta}_{0}, \ldots, \theta_{j-1}=\bar{\theta}_{j-1}$ and $\theta_{j}<\bar{\theta}_{j}$ for some integer $i \geq 0$. Finally, introducing $t$ as an undetermined variable and taking $\theta_{j}$ as the coefficients of a formal power series $\theta$ (invariant coordinate), we obtain $\theta=\theta_{0}+\theta_{1} t+\cdots=\sum_{j=0}^{\infty} \theta_{j} t^{j}$.

The sequences of symbols corresponding to periodic orbits of the critical points $c_{1}$ and $c_{2}$ are $P=A P_{1} P_{2} \ldots P_{p-1} A \ldots$ and $Q=B Q_{1} Q_{2} \ldots Q_{q-1} B \ldots$ In what follows we denote by $P^{(p)}=P_{1} P_{2} \ldots P_{p-1} A$ and $Q^{(q)}=Q_{1} Q_{2} \ldots Q_{q-1} B$ the periodic blocks associated to $P$ and $Q$. The realizable itineraries of the critical points $c_{1}$ and $c_{2}$ for the maps previously defined are called kneading sequences [10].

\section{Symbolic dynamics for symmetric bimodal maps}

Denote by $\mathscr{F}_{K S}$ the set of pairs of kneading sequences $(P, Q)$, with $(P, Q)$ either a pair of stable orbits or a doubly stable orbit. In Table 2.1, we give the subset of kneading sequences, with lengths $p, q<5$.

The corresponding columns are given by the conjugate of the previous sequences. 
Table 2.1. Kneading data for bimodal maps (detail). For the lines of the table, we have $1-R L L A$, 2 - RLA, 3-RLMA, 4-RLB, 5-RA, 6-RMRA, 7 - RMB, 8 - RMMA, 9 - RMMB, $10-R M A$, 11 - RMLB, 12 - RMLA, 13 - RB, 14 - RRLA, 15 - RRLB, 16 - RRA, 17 - RRMB, 18 - RRMA, 19 $R R B, 20-R R R A, 21-R R R B$

\begin{tabular}{|l|l|l|l|l|l|l|l|l|l|l|l|l|l|l|l|l|l|l|l|l|l|}
\hline & 1 & 2 & 3 & 4 & 5 & 6 & 7 & 8 & 9 & 10 & 11 & 12 & 13 & 14 & 15 & 16 & 17 & 18 & 19 & 20 & 21 \\
\hline 1 & & & & & & & & & & & & & & & & & & & & $*$ & \\
\hline 2 & & & & & & & & & & & & & & & & $*$ & & $*$ & & $*$ & \\
\hline 3 & & & & & & & & $*$ & & $*$ & & & & & & $*$ & & $*$ & & $*$ & \\
\hline 4 & & & & & & & $*$ & & $*$ & & & & $*$ & & & & $*$ & & $*$ & $*$ \\
\hline 5 & & & & & $*$ & $*$ & & $*$ & & $*$ & & & & & & $*$ & & $*$ & & $*$ & \\
\hline 6 & & & & & $*$ & $*$ & & $*$ & & $*$ & & & & & & $*$ & & $*$ & & $*$ & \\
\hline 7 & & & & $*$ & & & $*$ & & $*$ & & $*$ & & $*$ & & $*$ & & $*$ & & $*$ & $*$ \\
\hline 8 & & & $*$ & & $*$ & $*$ & & $*$ & & $*$ & & & & & & $*$ & & $*$ & & $*$ & \\
\hline 9 & & & & $*$ & & & $*$ & & $*$ & & $*$ & & $*$ & & $*$ & & $*$ & & $*$ & $*$ \\
\hline 10 & & $*$ & & $*$ & $*$ & & $*$ & & $*$ & & & & & & $*$ & & $*$ & & $*$ & $*$ \\
\hline 11 & & & & & & & $*$ & & $*$ & & $*$ & & $*$ & & $*$ & & $*$ & & $*$ & $*$ \\
\hline 12 & & & & & & & & & & & & & & & & $*$ & & $*$ & & $*$ & $*$ \\
\hline 13 & & & & $*$ & & & $*$ & & $*$ & & $*$ & & $*$ & & $*$ & & $*$ & & $*$ & $*$ \\
\hline 14 & & & & & & & & & & & & & & $\circledast$ & & $*$ & & $*$ & & $*$ & $*$ \\
\hline 15 & & & & & & & $*$ & & $*$ & & $*$ & & $*$ & & $\circledast$ & & $*$ & & $*$ & $*$ \\
\hline 16 & & $*$ & $*$ & & $*$ & $*$ & & $*$ & & $*$ & & $*$ & & $*$ & & $*$ & & $*$ & $*$ & $*$ \\
\hline 17 & & & & $*$ & & & $*$ & & $*$ & & $*$ & & $*$ & & $*$ & & $*$ & & $*$ & $*$ \\
\hline 18 & & $*$ & $*$ & & $*$ & $*$ & & $*$ & & $*$ & & $*$ & & $*$ & & $*$ & & $*$ & & $*$ & $*$ \\
\hline 19 & & & & $*$ & & & $*$ & & $*$ & & $*$ & & $*$ & & $*$ & & $*$ & & $*$ & $*$ \\
\hline 20 & $*$ & $*$ & $*$ & & $*$ & $*$ & & $*$ & & $*$ & & $*$ & & $*$ & & $*$ & & $*$ & & $*$ & $*$ \\
\hline 21 & & & & $*$ & & & $*$ & & $*$ & & $*$ & & $*$ & & $*$ & & $*$ & & $*$ & $*$ \\
\hline
\end{tabular}

We define a tree $\mathscr{D}$ that corresponds to the diagonal in $\mathscr{F}_{K S}$ and codify the symmetric bimodal maps. Each element $S \in \mathscr{D}$ is of one of the following types: $S$ is a pair of stable orbits, that is, $S=(P, \bar{P})=\left(P^{(p-1)} A, \overline{P^{(p-1)}} B\right)$; otherwise, $S$ is a doubly stable orbit, that is, $S=P^{(p-1)} B \overline{P^{(p-1)}} A$, where $\overline{P^{(p-1)}}=\overline{P_{1} P_{2}} \ldots \overline{P_{p-1}}$ with $\overline{P_{i}}=R$ if $P_{i}=L, \overline{P_{i}}=M$ if $P_{i}=M$, and $\overline{P_{i}}=L$ if $P_{i}=R$, and $1 \leq i \leq p-1$.

Note that the set $\mathscr{D}$ is ordered with respect to the order of the sequences $P$ (or the inverse order in $\bar{P}$ ) induced by the order of the symbols $-R<-B<-M<-A<-L<L<$ $A<M<B<R$.

Let $\mathscr{D}_{1}$ be a subset of $\mathscr{D}$ with elements between $\left(M^{\infty}, M^{\infty}\right)$ and $\left(R M^{\infty}, L M^{\infty}\right)$, see Figure 2.1. Let $S^{(2 p)}=\left(P^{(p-1)} A, \overline{P^{(p-1)}} B\right)$ or $S^{(2 p)}=P^{(p-1)} B \overline{P^{(p-1)}} A$ and consider a full tree $\mathcal{T}$ whose elements are also between $\left(M^{\infty}, M^{\infty}\right)$ and $\left(R M^{\infty}, L M^{\infty}\right)$ and are characterized by each vertex branch in two edges following the next rule.

Alternatively, the vertices in each level of the tree are doubly stable orbits $P^{(p-1)} B \overline{P^{(p-1)}} A$ or pairs of stable orbits $\left(P^{(p-1)} A, \overline{P^{(p-1)}} B\right)$. The doubly stable orbits occur in odd levels and the pairs of stable orbits in even levels. For the doubly stable orbit $P^{(p-1)} B \overline{P^{(p-1)}} A$ and according to whether the $M$-parity of $P^{(p-1)}$ is even or odd, the branching orders can be described, respectively, by 
72 Iterated symmetric bimodal maps

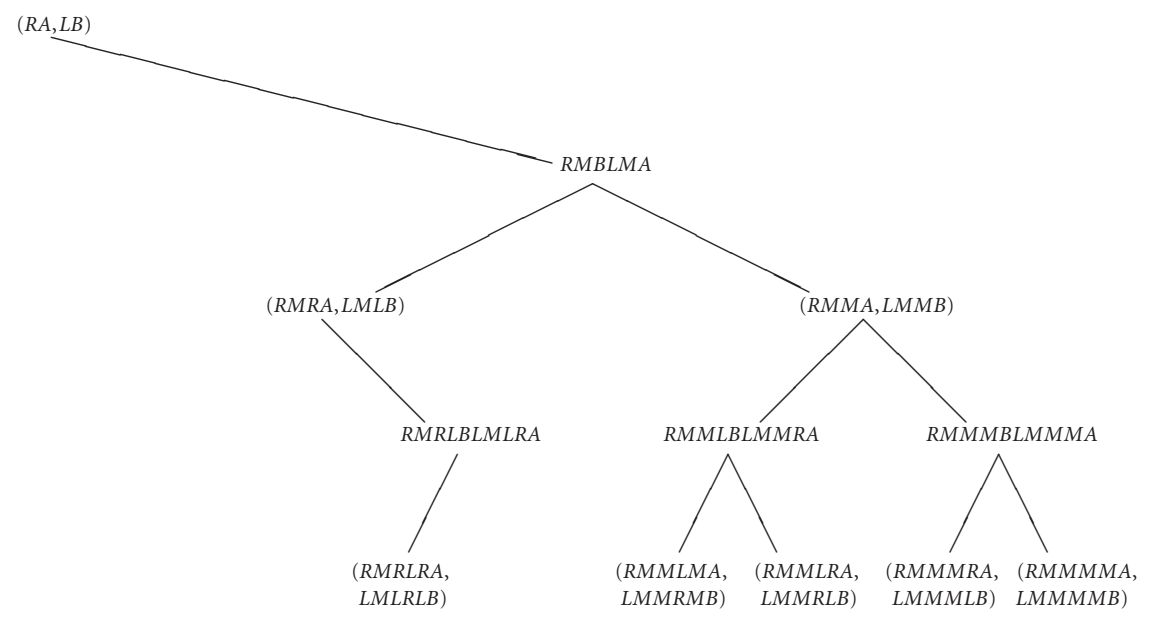

Figure 2.1. The tree $\mathscr{D}_{1}$.

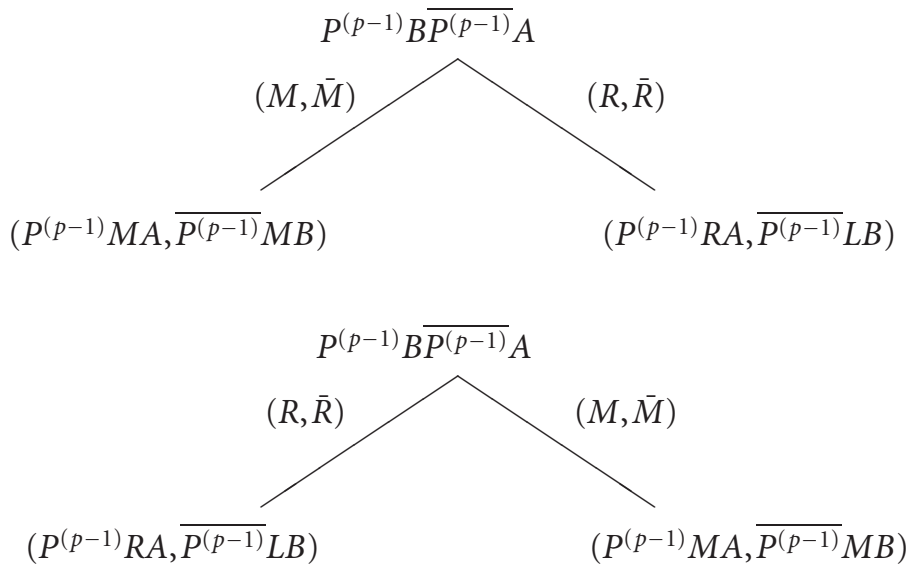

For the pairs of stable orbits, the branching orders can be described by

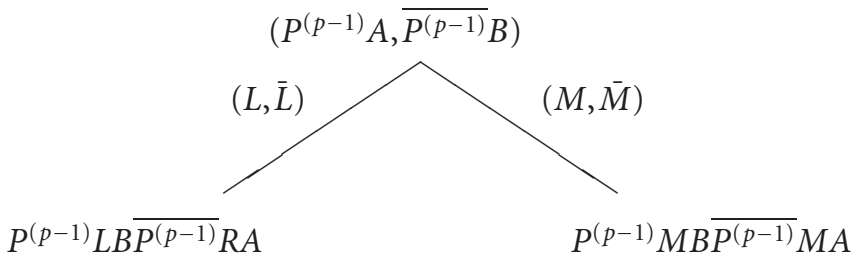




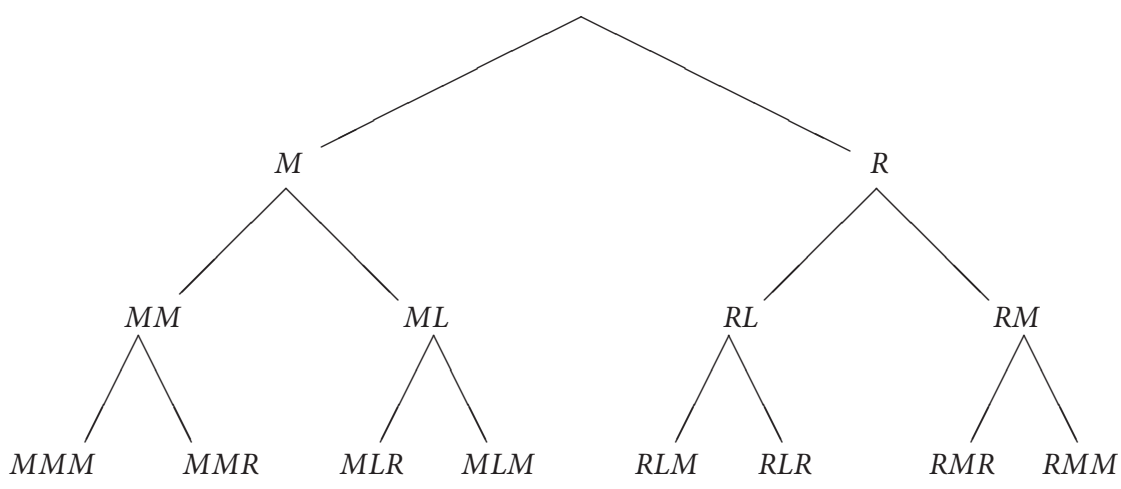

Figure 2.2. The tree $\mathscr{T}$.

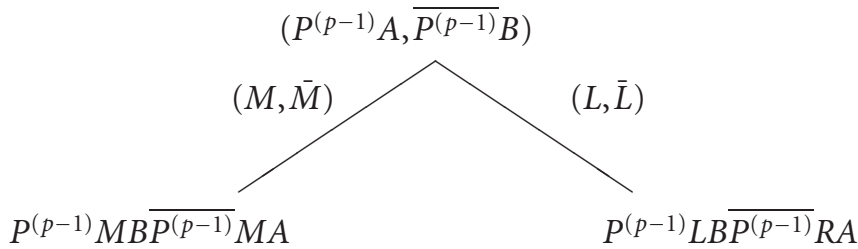

according to whether the $M$-parity of $P^{(p-1)} A$ is even or odd, respectively.

Using these rules, we get, as mentioned before, the full tree $\mathscr{T}$, see Figure 2.2. The next result establishes that to each $S=\left(P^{(p-1)} A, \overline{P^{(p-1)}} B\right)$ or $P^{(p-1)} B \overline{P^{(p-1)}} A$ in $\mathscr{D}_{1}$ corresponds a sequence $P^{(p-1)}$ in $\mathscr{T}$.

Lemma 2.1. If $S \in \mathscr{D}_{1}$, then $P^{(p-1)} \in \mathcal{T}$.

Proof. Let $S=P^{(p-1)} B \overline{P^{(p-1)}} A \in \mathscr{D}_{1}$ be a doubly stable orbit (odd level), with odd $M$ parity. Then, we have

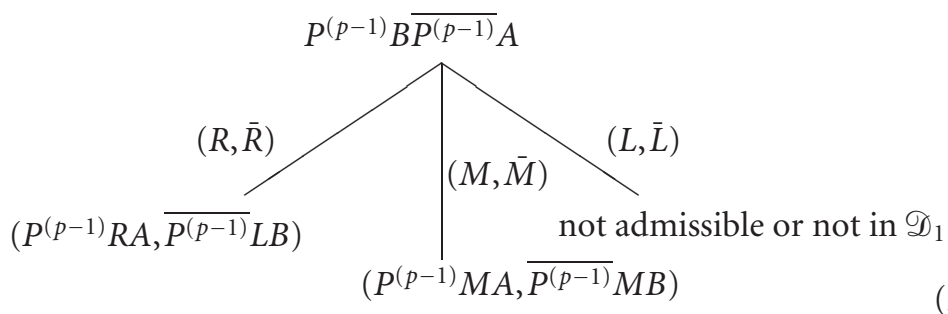


The doubly stable orbit $P^{(p-1)} B \overline{P^{(p-1)}} A$ leads, on the next level, to the pairs of stable orbits given by $\left(P^{(p-1)} X A, \overline{P^{(p-1)}} \bar{X} B\right)=(R M \ldots X A, L M \ldots \bar{X} B)$. Note that when $(X, \bar{X})=(L, \bar{L})$,

$$
\sigma^{(p-1)}(L M \ldots \bar{X} B)=\sigma^{(p-1)}(L M \ldots R B)=R B \ldots>R M \ldots
$$

which is not admissible or is not in $\mathscr{D}_{1}$. In the same way, the doubly stable ones obtained from pairs of stable orbits follow the rule in $\mathscr{T}$ because now the branch associated to $(R, \bar{R})$ is not admissible. The proof for the case when the $M$-parity of $P^{(p-1)} A$ is even is analogous.

In what follows, we denote by $\mathscr{T}_{K S}$ the set of kneading sequences associated to unimodal maps. Then, we have the following theorem.

Theorem 2.2. The tree $\mathscr{D}_{1}$ is isomorphic to $\mathscr{T}_{K S}$.

Proof. Let $\mathscr{E}$ be a complete tree with two symbols $\{L, R\}$, where we consider the $R$-parity. There exists an isomorphism between $\mathscr{T}$ and $\mathscr{E}$, where each symbol $L$ in $\mathscr{E}$ corresponds to a symbol $M$ in $\mathscr{T}$ and each symbol $R$ in $\mathscr{E}$ corresponds to a symbol $L$ or $R$ in $\mathscr{T}$ according to whether the $(k-1)$-level is even or odd, respectively. Thus the $R$-parity in $\mathscr{E}$ corresponds to the $(R+L)$-parity in $\mathscr{T}$, and so we have

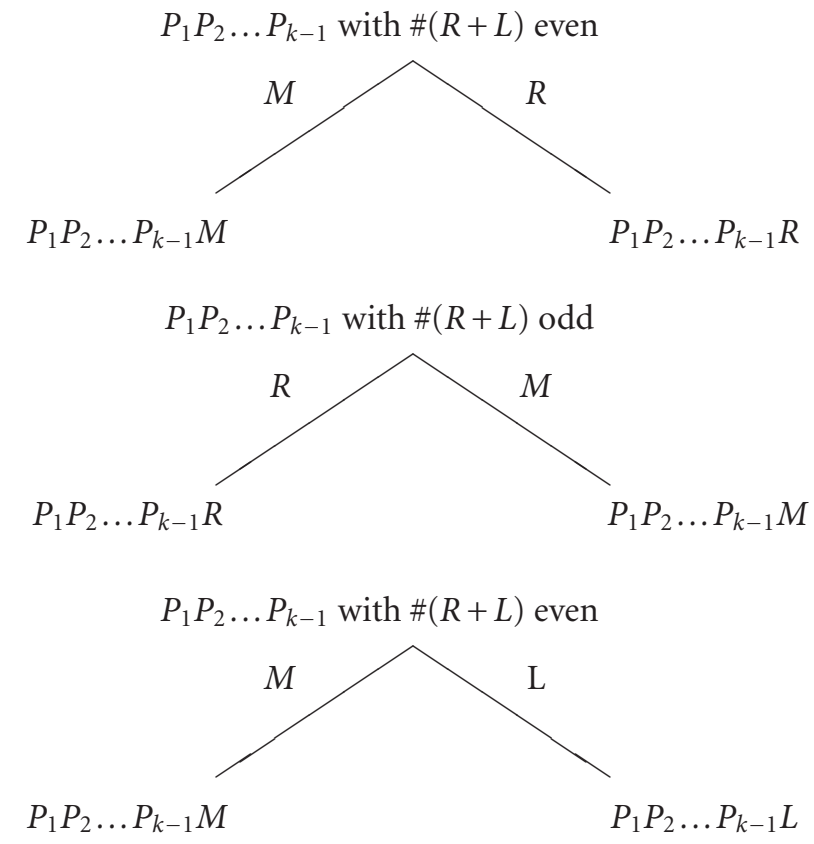


if the $(k-1)$-level is even, and

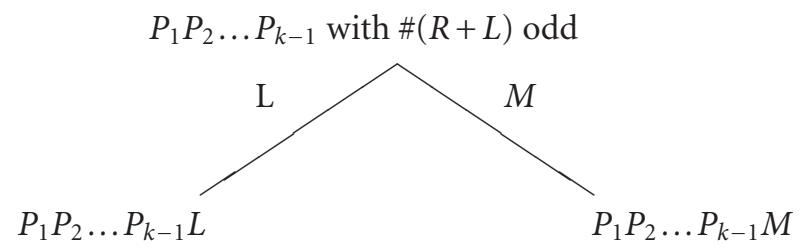

if the $(k-1)$-level is odd. To each admissible vertex $P^{(p-1)} C$, when we join $C$ to the end of a block $P^{(p-1)}$ in $\mathscr{E}$, corresponds the symbol $A$ (or $B$ ) in the even or odd level in $\mathscr{T}$. Thus, to each admissible vertex $P^{(p-1)} C$ in $\mathscr{T}_{K S}$ corresponds an admissible vertex $\left(\tilde{P}^{(p-1)} A, \widetilde{P}^{(p-1)} B\right)$ or $\tilde{P}^{(p-1)} B \bar{P}^{(p-1)} A$ in $\mathscr{D}_{1}$. Note that the admissibility in $\mathscr{E}$ corresponds to the admissibility in $\mathscr{T}$ since the $R$-parity in $\mathscr{E}$ corresponds to the $(R+L)$-parity in $\mathcal{T}$ and the shift $\sigma$ acting on $P^{(p-1)} C$ corresponds in $\mathscr{T}$ to a shift $\sigma$ acting on $\tilde{P}^{(p-1)} A$ or $\tilde{P}^{(p-1)} B \bar{P}^{(p-1)} A$. In this way, if $P^{(p-1)} C$ is admissible, that is,

$$
\sigma^{i}\left(P^{(p-1)} C\right) \leq P^{(p-1)} C, \quad \forall i
$$

then we also have

$$
\sigma^{i}\left(\tilde{P}^{(p-1)} A\right) \leq \tilde{P}^{(p-1)} A, \quad \forall i
$$

or

$$
\sigma^{i}\left(\tilde{P}^{(p-1)} B \bar{P}^{(p-1)} A\right) \leq \tilde{P}^{(p-1)} B \bar{P}^{(p-1)} A, \quad \forall i .
$$

Consider now the Markov matrix associated to a sequence $S=\tilde{P}^{(p-1)} B \overline{P^{(p-1)}} A$ or $\left(\tilde{P}^{(p-1)} A, \overline{P^{(p-1)}} B\right)$ and denote by $d_{P}(t)$ the characteristic polynomial of the Markov matrix $A_{P}$, where $P$ equals $P^{(p-1)} C \in \mathscr{T}_{K S}$ and corresponds to $\tilde{P}=\tilde{P}^{(p-1)} X$ in $\mathscr{D}_{1}$, where $X=A$ or $B$. Then the following result holds.

Proposition 2.3. For each $S=\tilde{P}^{(p-1)} B \overline{P^{(p-1)}} A$ or $\left(\tilde{P}^{(p-1)} A, \overline{P^{(p-1)}} B\right) \in \mathscr{D}_{1}$, there exists a decomposition of the matrix $A_{S}$ of type

$$
A_{S}=\left[\begin{array}{ccc}
1 & W_{1} & W_{2} \\
0 & 0 & A_{P} \\
0 & A_{P} & 0
\end{array}\right] .
$$


Proof. Let $S=\tilde{P}^{(p-1)} B \tilde{\bar{P}}^{(p-1)} A$ or $\left(\tilde{P}^{(p-1)} A, \tilde{\bar{P}}^{(p-1)} B\right) \in \mathscr{D}_{1}$; then the Markov partition associated to $S$ is given by $\mathscr{P}=\mathscr{P}_{1} \cup \mathscr{P}_{2} \cup \mathscr{P}_{3}$, where $\mathscr{P}_{1}=\left\{I_{i}: 1 \leq i \leq p-1\right\}, \mathscr{P}_{2}=I_{p}$, $\mathscr{P}_{3}=\left\{I_{i}: p+1 \leq i \leq 2 p-1\right\}$, and $\partial I_{i}=z_{i+1}-z_{i}$. When $S=\left(\tilde{P}^{(p-1)} B \tilde{\bar{P}}^{(p-1)} A\right)$, we have

$$
z_{i} \in J_{1}=\left\{x_{2 j}: 0 \leq j<p\right\} \quad \text { if } I_{i} \in \mathscr{P}_{1},
$$

or

$$
z_{i} \in J_{2}=\left\{x_{2 j+1}: 0 \leq j<p\right\} \quad \text { if } I_{i} \in \mathscr{P}_{3}
$$

where $x_{0}$ (resp., $x_{p}$ ) corresponds to the critical point $c_{1}$ (resp., $c_{2}$ ). On the other hand, if $S=\left(\tilde{P}^{(p-1)} A, \tilde{\bar{P}}^{(p-1)} B\right)$, then

$$
z_{i} \in J_{3}=\left\{x_{2 j}, y_{2 j}: 0 \leq j<\frac{p-2}{2}\right\} \quad \text { if } I_{i} \in \mathscr{P}_{1},
$$

or

$$
z_{i} \in J_{4}=\left\{x_{2 j+1}, y_{2 j+1}: 0 \leq j<\frac{p-2}{2}\right\} \quad \text { if } I_{i} \in \mathscr{P}_{3},
$$

and, in both cases, $\mathscr{P}_{2}=\left\{I_{p}\right\}$, with $\partial I_{p}=z_{p+1}-z_{p}$, where $z_{p}=\max \left\{J_{1}\left(\right.\right.$ or $\left.\left.J_{3}\right)\right\}$ and $z_{p+1}=$ $\min \left\{J_{2}\left(\right.\right.$ or $\left.\left.J_{4}\right)\right\}$. Note also that if we look for the structure of $\mathscr{D}_{1}$, we will conclude that if $S=\tilde{P}^{(p-1)} B \bar{P}^{(p-1)} A \in \mathscr{D}_{1}$, then $S_{2 i} \in\{L, A, M\}, S_{2 i+1} \in\{M, B, R\}$ with $0 \leq i<p$. If $S=\left(\tilde{P}^{(p-1)} A, \bar{P}^{(p-1)} B\right) \in \mathscr{D}_{1}$, then $\tilde{P}_{2 i} \in\{L, M\}, \tilde{P}_{2 i+1} \in\{M, R\}, \bar{P}_{2 i} \in\{M, R\}$, and $\bar{P}_{2 i+1} \in$ $\{L, M\}$ for $0 \leq i \leq(p-2) / 2$. Thus, the even points establish a Markov shift and the odd points establish another Markov shift that is isomorphic to the previous one according to the symmetry of the cubic (where $S \in \mathscr{D}_{1}$ ). So, we only have to prove that each one of these shifts is isomorphic to the unimodal map associated with $\left(A_{P^{(p)}}, \sigma_{A}\right)$. Note that the even points are all less than the fixed point (that corresponds to the sequence of symbols $M^{\infty}$ ), whereas the odd points are all higher than the fixed point. So, we get two unimodal maps with critical points $c_{1}$ and $c_{2}$ given by sequences of symbols in $\{L, A, M\}$ or $\{M, B, R\}$ and by the admissibility unimodal rules. Thus, the partitions $\mathscr{P}_{1}$ and $\mathscr{P}_{3}$ are equivalent to the unimodal map associated, and so they have the same Markov shifts. Finally, $\mathscr{P}_{2}$ introduces a state that is transit to itself and also has transitions for other states that correspond to the transient part of the dynamics, $W$.

Corollary 2.4. For each $S=\tilde{P}^{(p-1)} B \overline{P^{(p-1)}} A$ or $\left(\tilde{P}^{(p-1)} A, \overline{P^{(p-1)}} B\right) \in \mathscr{D}_{1}$, there exists a decomposition of the characteristic polynomial, $d_{S}(t)=\operatorname{det}\left(I-t \cdot A_{S}\right)$, associated to $A_{S}$, and it is given by

$$
d_{S}(t)=(1-t) d_{P}(t) d_{P}(-t)
$$

where $d_{P}(t)=\operatorname{det}\left(I-t \cdot A_{P}\right)$.

Proof. Note that the decomposition of the characteristic polynomial follows from the previous decomposition of the Markov matrix. 


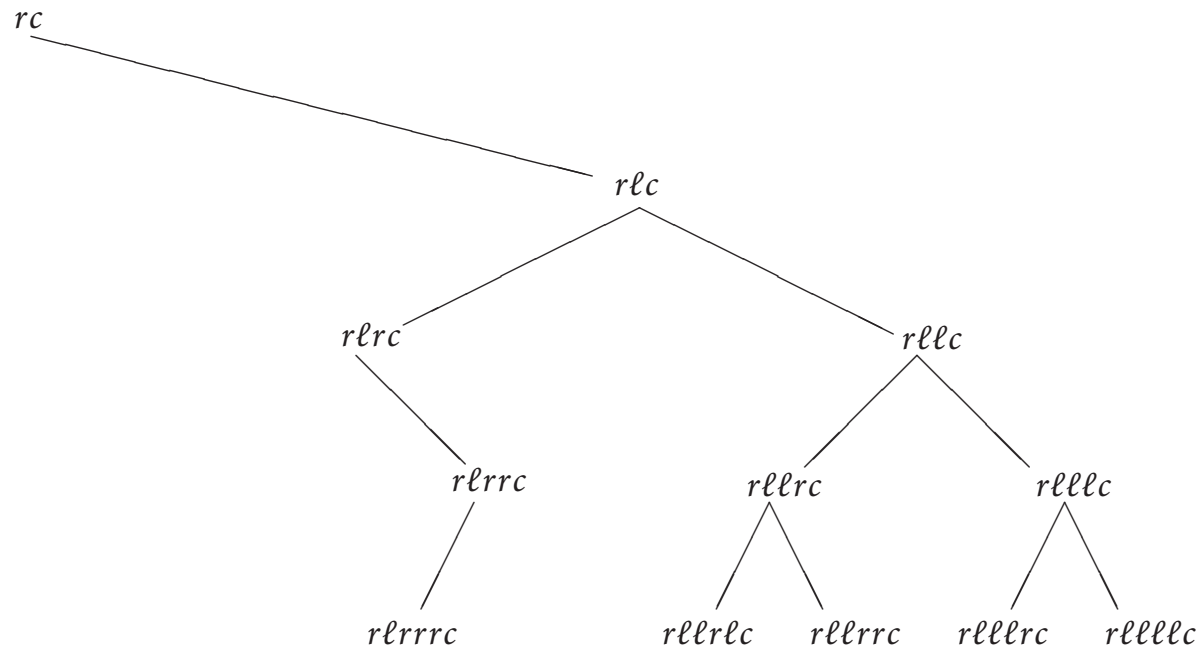

Figure 3.1. The tree $\mathcal{U}$ of unimodal kneading sequences.

\section{3. *-product operator}

For the unimodal case, the $*$-product operator of symbolic sequences was defined (see [4]). This product turns out to be a very useful tool to understand the properties of such maps.

In what follows, we extend the $*$-product operator for the case of symbolic sequences associated to symmetric bimodal maps. Note that in $\mathscr{D}$ the $*$-operation is consistent with the initial definition of the $*$-product introduced by Derrida, Gervois, and Pomeau for the unimodal case (see also $[8,9,11]$ for the bimodal case).

According to Theorem 2.2, the tree $\mathscr{D}_{1}$ is isomorphic to $\mathcal{U}$ (ordered set of unimodal kneading sequences, see Figure 3.1).

From this set we can define another tree $\mathscr{F}^{+}=\left\{\mathscr{F}^{-}=\mathcal{U} u\right.$, if the level is odd, or $\mathscr{F}^{+}=$ $(\mathcal{U}, \sigma(\mathcal{U}))$ if the level is even\}, see Figure 3.2. Now, using the symbolic codification applied to $f \circ f$, with $f$ a unimodal map, we introduce the following translation rules:

$$
\begin{gathered}
\ell \ell \longrightarrow L, \quad \ell c \longrightarrow A, \quad \ell r \longrightarrow M, \quad c r \longrightarrow B, \\
\quad r r \rightarrow R, \quad r c \longrightarrow C, \quad r \ell \longrightarrow U .
\end{gathered}
$$

By applying these rules, Figure 3.2 can be rewritten as the tree $\mathscr{G}_{=} \mathscr{G}^{+} \cup \mathscr{G}^{-}$, see Figure 3.3.

Remark 3.1. Let $\left(x_{1} \ldots x_{2 n-1} c, \sigma\left(x_{1} \ldots x_{2 n-1} c\right)\right) \in \mathscr{F}^{+}$or $x_{1} \ldots x_{2 n} c x_{1} \ldots x_{2 n} c \in \mathscr{F}^{-}$, where $x_{i} \in\{\ell, r\}$ and $\mathscr{F}=\mathscr{F}^{+} \cup \mathscr{F}^{-}$is the tree presented in Figure 3.2. Let 2 be the set of trimodal kneading data such that the images of both maxima are equal. With $D=A$ 
78 Iterated symmetric bimodal maps

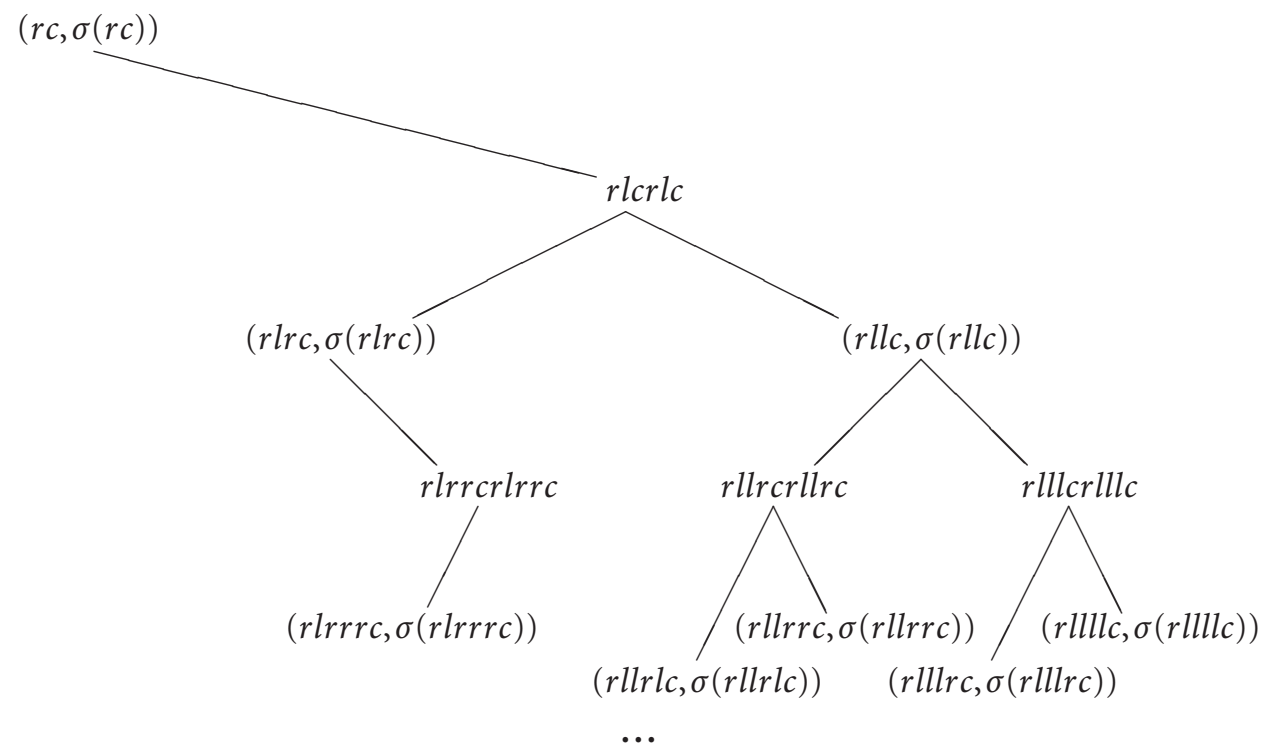

Figure 3.2. The tree $\mathscr{F}$.

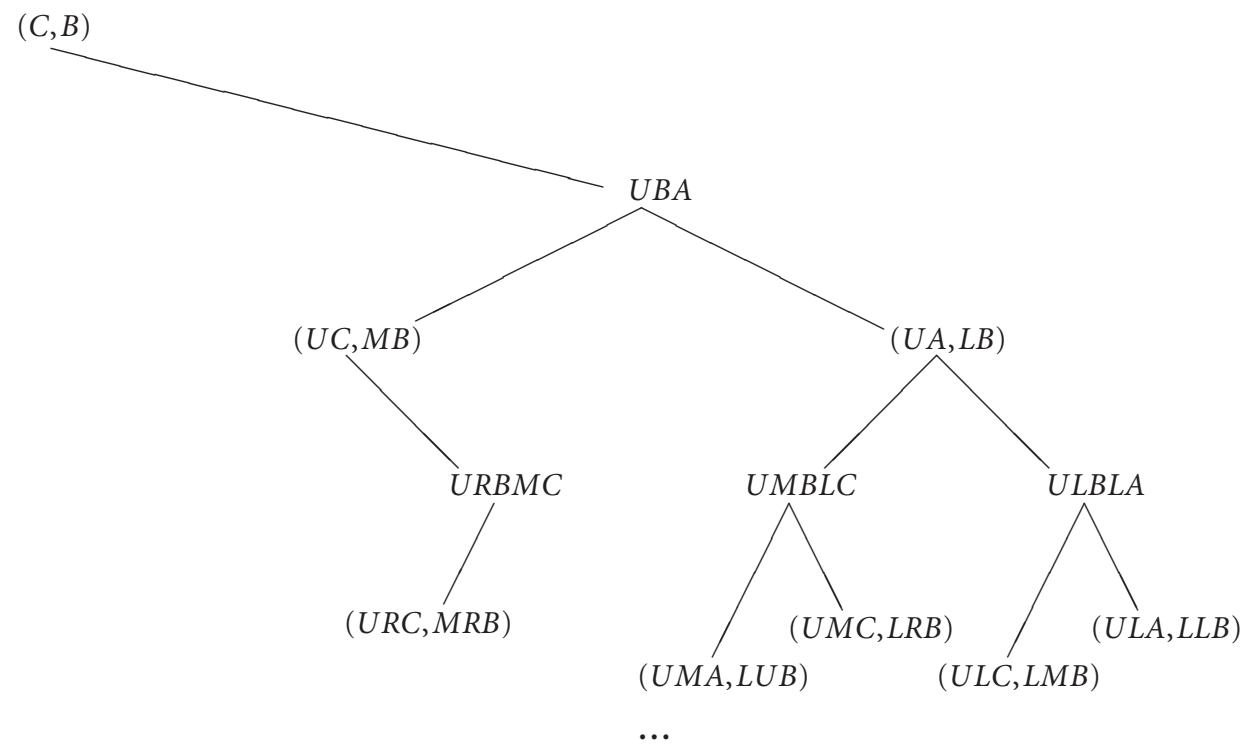

Figure 3.3. The tree $\mathscr{G}$ of the second factor for the $*$-product.

or $B$, we will write $\left(P_{1} \ldots P_{2 p-1} D, Q_{1} \ldots Q_{2 p-1} B, P_{1} \ldots P_{2 p-1} D\right) \in 2$, for even levels, and $\left(P_{1} \ldots P_{2 p-1} B Q_{1} \ldots Q_{2 p-2} A, P_{1} \ldots P_{2 p-1} B\right) \in 2$, for odd levels, with $P_{i}, Q_{j} \in\{L, A, M, B, R$, $C, U\}=\{\ell \ell, \ell c, \ell r, c r, r r, r c, r \ell\}$. With this notation, we can get $\mathscr{G}$ from the tree $\mathscr{F}_{\text {f }}$ (see also [6]). 
Thus, we will consider the following different situations for the definition of the *-product: first, let $F=(P, \bar{P}) \in \mathscr{D}$ and $G=\left(x_{1} x_{2} \ldots x_{x-1} c, x_{1} x_{2} \ldots x_{x-1} c\right)$, with $x_{1} x_{2} \ldots$ $x_{x-1} c \in \mathcal{U}$; then, we let $F=P B \bar{P} A \in \mathscr{D}$ and $G \in \mathscr{G}$.

Type 1. Let $F=(P, \bar{P})=\left(P^{(p-1)} A, \overline{P^{(p-1)}} B\right) \in \mathscr{D}$ be a bimodal kneading datum and $G=$ $(X, X)$ a pair of unimodal kneading sequences. Then we have

$$
F * G=(P, \bar{P}) *(X, X)=\left(P^{(p-1)} * X^{(x-1)} c, \overline{P^{(p-1)}} * X^{(x-1)} c\right)
$$

with

$$
P^{(p-1)} * X^{(x-1)} c=P^{(p-1)} A_{1}^{ \pm} P^{(p-1)} A_{2}^{ \pm} \ldots P_{x-1}^{(p-1)} A_{x-1}^{ \pm} P^{(p-1)} A
$$

where

$$
A_{i}^{ \pm}=\left\{\begin{array}{ll}
M & \text { if } x_{i}=r, \\
A & \text { if } x_{i}=c, \\
L & \text { if } x_{i}=\ell,
\end{array} \quad \text { if } P \text { is even, } \quad A_{i}^{ \pm}=\left\{\begin{array}{ll}
L & \text { if } x_{i}=r, \\
A & \text { if } x_{i}=c, \\
M & \text { if } x_{i}=\ell,
\end{array} \quad \text { if } P \text { is odd } .\right.\right.
$$

In a similar way,

$$
\overline{P^{(p-1)}} * X^{(x-1)} c=\overline{P^{(p-1)}} B_{1}^{ \pm} \overline{P^{(p-1)}} B_{2}^{ \pm} \ldots \overline{P^{(p-1)}} B_{y-1}^{ \pm} \overline{P^{(p-1)}} B
$$

where

$$
B_{i}^{ \pm}=\left\{\begin{array}{ll}
M & \text { if } x_{i}=r, \\
B & \text { if } x_{i}=c, \\
R & \text { if } x_{i}=\ell,
\end{array} \quad \text { if } \bar{P} \text { is even, } \quad B_{i}^{ \pm}=\left\{\begin{array}{ll}
R & \text { if } x_{i}=r, \\
B & \text { if } x_{i}=c, \\
M & \text { if } x_{i}=\ell,
\end{array} \text { if } \bar{P}\right. \text { is odd }\right.
$$

Type 2. Let $F=P B \bar{P} A=P^{(p-1)} B \overline{P^{(p-1)}} A \in \mathscr{D}$ and $G=X^{(x-1)} B Y^{(y-1)} D \in G^{-}$(where $D=$ $A$ or $C$ ) be two kneading data. Then

$$
\begin{aligned}
F * G= & P^{(p-1)} B_{1}^{ \pm} \overline{P^{(p-1)}} A_{1}^{ \pm} P^{(p-1)} B_{2}^{ \pm} \overline{P^{(p-1)}} A_{2}^{ \pm} \ldots P^{(p-1)} B \overline{P^{(p-1)}} A_{x}^{ \pm} P^{(p-1)} \\
& \times B_{x+1}^{ \pm} \overline{P^{(p-1)}} A_{x+1}^{ \pm} P^{(p-1)} B_{x+2}^{ \pm} \ldots \overline{P^{(p-1)}} A_{x+y-1}^{ \pm} P^{(p-1)} B_{x+y}^{ \pm} \overline{P^{(p-1)}} A .
\end{aligned}
$$


Let $Z_{i}=X_{i}, Z_{x+i}=Y_{i}$, and $Z_{x+y}=D$; then

$$
P^{(p-1)} B_{i}^{ \pm} \overline{P^{(p-1)}} A_{i}^{ \pm}= \begin{cases}P^{(p-1)} M \overline{P^{(p-1)}} M & \text { if } Z_{i}=L, \\
P^{(p-1)} M \overline{P^{(p-1)}} A & \text { if } Z_{i}=A, \\
P^{(p-1)} M \overline{P^{(p-1)}} L & \text { if } Z_{i}=M, \\
P^{(p-1)} B \overline{P^{(p-1)}} L & \text { if } Z_{i}=B, \\
P^{(p-1)} R \overline{P^{(p-1)}} L & \text { if } Z_{i}=R, \\
P^{(p-1)} R \overline{P^{(p-1)}} A & \text { if } Z_{i}=C, \\
P^{(p-1)} R \overline{P^{(p-1)}} M & \text { if } Z_{i}=U, \\
B_{i}^{ \pm} \overline{P^{(p-1)}} A_{i}^{ \pm}=\left\{\begin{array}{ll}
P^{(p-1)} R \overline{P^{(p-1)}} L & \text { if } Z_{i}=L, \\
P^{(p-1)} R \overline{P^{(p-1)}} A & \text { if } Z_{i}=A, \\
P^{(p-1)} R \overline{P^{(p-1)}} M & \text { if } Z_{i}=M, \\
P^{(p-1)} B \overline{P^{(p-1)}} M & \text { if } Z_{i}=B, \\
P^{(p-1)} M \overline{P^{(p-1)}} M & \text { if } Z_{i}=R, \\
P^{(p-1)} M \overline{P^{(p-1)}} A & \text { if } Z_{i}=C, \\
P^{(p-1)} M \overline{P^{(p-1)}} L & \text { if } Z_{i}=U,
\end{array} \text { if } P \text { and } \bar{P}\right. \text { are odd. }\end{cases}
$$

Type 3. Let $F=P^{(p-1)} B \overline{P^{(p-1)}} A \in \mathscr{D}$ and $G=\left(X^{(n-1)} D, Y^{(n-1)} B\right) \in \mathscr{G}^{+}$be two kneading data. Then

$$
\begin{aligned}
F * G= & \left(P^{(p-1)} B_{1}^{ \pm} \overline{P^{(p-1)}} A_{1}^{ \pm} P^{(p-1)} B_{2}^{ \pm} \overline{P^{(p-1)}} A_{2}^{ \pm} \ldots P^{(p-1)} B_{n}^{ \pm} \overline{P^{(p-1)}} A,\right. \\
& \left.\overline{P^{(p-1)}} A_{n+1}^{ \pm} P^{(p-1)} B_{n+1}^{ \pm} \overline{P^{(p-1)}} A_{n+2}^{ \pm} P^{(p-1)} B_{n+2}^{ \pm} \ldots \overline{P^{(p-1)}} A_{2 n}^{ \pm} P^{(p-1)} B\right) .
\end{aligned}
$$

The transformation rules are the same as above for the first sequence

$$
F * X^{(x-1)} A=P^{(p-1)} B_{1}^{ \pm} \overline{P^{(p-1)}} A_{1}^{ \pm} P^{(p-1)} B_{2}^{ \pm} \overline{P^{(p-1)}} A_{2}^{ \pm} \ldots P^{(p-1)} B_{n}^{ \pm} \overline{P^{(p-1)}} A,
$$

except that $Z_{i}=B$ cannot occur. For the second position of the pair, we have

$$
\begin{aligned}
& \sigma^{p-1}(F) * \sigma^{n-1}\left(Y^{(n-1)} B\right) \\
& \quad=B \overline{P^{(p-1)}} A_{n+1}^{ \pm} P^{(p-1)} B_{n+1}^{ \pm} \overline{P^{(p-1)}} A_{n+2}^{ \pm} P^{(p-1)} B_{n+2}^{ \pm} \ldots \overline{P^{(p-1)}} A_{2 n}^{ \pm} P^{(p-1)}, \\
& \sigma\left(\sigma^{p-1}(F) * \sigma^{n-1}\left(Y^{(n-1)} B\right)\right) \\
& \quad=\overline{P^{(p-1)}} A_{n+1}^{ \pm} P^{(p-1)} B_{n+1}^{ \pm} \overline{P^{(p-1)}} A_{n+2}^{ \pm} P^{(p-1)} B_{n+2}^{ \pm} \ldots \overline{P^{(p-1)}} A_{2 n}^{ \pm} P^{(p-1)} B,
\end{aligned}
$$


where

$$
\begin{aligned}
B_{i}^{ \pm} \overline{P^{(p-1)}} A_{i}^{ \pm} P^{(p-1)}=\left\{\begin{array}{ll}
M \overline{P^{(p-1)}} M P^{(p-1)} & \text { if } Y_{i}=L, \\
M \overline{P^{(p-1)}} L P^{(p-1)} & \text { if } Y_{i}=M, \\
B \overline{P^{(p-1)}} L P^{(p-1)} & \text { if } Y_{i}=B, \\
R \overline{P^{(p-1)}} L P^{(p-1)} & \text { if } Y_{i}=R, \\
R \overline{P^{(p-1)}} M P^{(p-1)} & \text { if } Y_{i}=U,
\end{array} \quad \text { if } P \text { and } \bar{P}\right. \text { are even, } \\
B_{i}^{ \pm} \overline{P^{(p-1)}} A_{i}^{ \pm} P^{(p-1)}= \begin{cases}R \overline{P^{(p-1)}} L P^{(p-1)} & \text { if } Y_{i}=L, \\
R \overline{P^{(p-1)}} M P^{(p-1)} & \text { if } Y_{i}=M, \\
B \overline{P^{(p-1)}} M P^{(p-1)} & \text { if } Y_{i}=B, \\
M \overline{P^{(p-1)}} M P^{(p-1)} & \text { if } Y_{i}=R, \\
M \overline{P^{(p-1)}} L P^{(p-1)} & \text { if } Y_{i}=U,\end{cases}
\end{aligned}
$$

The following examples illustrate the definitions given above.

Example 3.2.

$(R M M A, L M M B) *(r \ell c, r \ell c)=(R M M M R M M L R M M A, L M M M L M M R L M M B)$.

Example 3.3.

$$
R B L A * U L B L A=R R L M R M L M R B L L R M L M R M L A .
$$

Example 3.4.

$$
R B L A *(U A, L B)=(R R L M R M L A, L L R M L M R B) .
$$

Remark 3.5. Notice that, regarding Example 3, where both $P$ and $\bar{P}$ are even sequences, we have, for the second position of the pair,

$$
\sigma(\sigma(R B L A) * \sigma(L B))=\sigma(B L A R * B L)=\sigma(B L L R M L M R)=L L R M L M R B .
$$

Remark 3.6. The $*$-product in $\mathscr{D}$ is not a true binary product $A * B=C$, for all $A$ and $B \in \mathscr{D}$. When $C$ is factorizable, with $C \in \mathscr{D}$, then $B$ must be in $\mathscr{G}$.

Remark 3.7. Note that for all $A \in \mathscr{D}$ and $B \in \mathscr{G}$, the result of the $*$-product defined previously is also in $\mathscr{D}$. 


\section{4. $\otimes$-product between Markov matrices}

In the same way, we can extend the $\otimes$-product between Markov matrices (introduced for unimodal maps in [5]) associated to symmetric bimodal maps, that is,

$$
A_{S}=A_{V} \otimes A_{W}
$$

where $S=V * W$ with $V \in \mathscr{D}$ and $W \in \mathscr{G}$.

Theorem 4.1. Let $V \in \mathscr{D}$ and $W \in \mathscr{G}$; then there exists a matrix product such that

$$
A_{S}=A_{V * W}=A_{V} \otimes A_{W}
$$

Proof. The proof is based on a construction of a product on the matrices induced by the *-product between kneading sequences. We will give this construction but only for the $*$-product between kneading sequences of the first type. For the others, it is technically similar and can be reproduced from this one. Let $W=\left(x_{1} x_{2} \ldots x_{k-1} c, x_{1} x_{2} \ldots x_{k-1} c\right) \in \mathscr{G}$. First of all, note that the matrix $A_{W}$ is symmetric, and so it can be written in the form

$$
A_{W}=\left[\begin{array}{ccc}
0 & 0 & \widehat{B}_{X} \\
N_{1} & 1 & N_{2} \\
B_{X} & 0 & 0
\end{array}\right],
$$

where $\left[\begin{array}{lll}N_{1} & 1 & N_{2}\end{array}\right]$ is the $k$ th row and $\left[\begin{array}{lll}0 & 1 & 0\end{array}\right]$ is the $k$ th column. Denoting $B_{X}=$ $\left[b_{i j}\right]$, then we define the $(k-1) \times(k-1)$ matrix $\hat{B}_{X}$ by $\hat{B}_{X}=B_{X}$ if $P^{(p)}$ is even and by $\hat{B}_{X}=\left[\hat{b}_{m j}\right]$ with $\hat{b}_{m j}=b_{i j}$, where $m=k-i$, if $P^{(p)}$ is odd. Given $V=(P, \bar{P}) \in \mathscr{D}$ and $W=(X, \bar{X}) \in \mathscr{G}$, it is immediate that their associated transition matrices, $A_{V}$ and $A_{W}$, are square, $(2 p-1)$ - and $(2 k-1)$-dimensional matrices, respectively, where $p$ and $k$ denote the numbers of symbols of the sequences $P$ and $X$. Analogously, it is fairly simple to see that the transition matrix associated with the sequence $V * W$ is a square matrix with dimension $(2 p k-1)$. Now we need to show that the elements of $A_{V * W}$ are completely determined by knowing the matrices $A_{V}$ and $A_{W}$. Consider the symbolic shifts of the sequences $P * X$ and $\bar{P} * \bar{X}$ and denote the corresponding points of the interval by $p_{i}^{j}$ and $q_{i}^{j}$, that is, $p_{i}^{j}$ will be the point corresponding to the sequence $\sigma^{p(j-1)+(i-1)}(P * X)$ and $q_{i}^{j}$ the point corresponding to the sequence $\sigma^{p(j-1)+(i-1)}(\bar{P} * \bar{X})$. When one considers the collection of points of the interval from all the shifts cited above, one can see that they appear as groups of blocks of $x$ points. Considering the order of the shifted sequences $\sigma^{i}(P), \sigma^{j}(\bar{P}), \sigma^{k}(X)$, and $\sigma^{n}(\bar{X})$ and the way those sequences appear as subsets of the partition induced by the sequence $V * W$, we can conclude (see also [5]) that the matrix 
$A_{V * W}$ has the following block structure:

$$
\left[\begin{array}{ccccc}
A_{1,1} & A_{1,2} & \cdots & A_{1, l+m+r} & N_{1} \\
\vdots & \vdots & & \vdots & \vdots \\
A_{l, 1} & A_{l, 2} & \cdots & A_{l, l+m+r} & N_{l} \\
Z_{l+1,1} & Z_{l+1,2} & \cdots & Z_{l+1, l+m+r} & \bar{A}_{X} \\
A_{l+2,1} & A_{l+2,2} & \cdots & A_{l+2, l+m+r} & N_{l+2} \\
\vdots & \vdots & & \vdots & \vdots \\
A_{l+m+1,1} & A_{l+m+1,2} & \cdots & A_{l+m+1, l+m+r} & N_{l+m+1} \\
\tilde{A}_{X} & Z_{l+m+2,2} & \cdots & Z_{l+m+2, l+m+r} & N_{l+m+2} \\
N_{l+m+3} & A_{l+m+3,2} & \cdots & A_{l+m+3, l+m+r} & A_{l+m+3, l+m+r+1} \\
\vdots & \vdots & & \vdots & \vdots \\
N_{l+m+r+2} & A_{l+m+r+2,2} & \cdots & A_{l+m+r+2, l+m+r} & A_{l+m+r+2, l+m+r+1}
\end{array}\right] \text {, }
$$

where $l, m$, and $r$ are, respectively, the numbers of the symbols $L, M$, and $R$ in the sequence $V, A_{i, j}$, with $(i, j) \neq(l+m+1,1)$, is either one of the $k \times k$ matrices

$$
\begin{aligned}
& {\left[\begin{array}{cccc}
1 & 1 & \cdots & 1 \\
0 & 0 & \cdots & 0 \\
\vdots & \vdots & & \vdots \\
0 & 0 & \cdots & 0
\end{array}\right],} \\
& {\left[\begin{array}{cccc}
0 & \cdots & 0 & 1 \\
0 & \cdots & 1 & 0 \\
\vdots & & \vdots & \vdots \\
1 & \cdots & 0 & 0
\end{array}\right], \quad\left[\begin{array}{cccc}
1 & 0 & \cdots & 0 \\
0 & 1 & \cdots & 0 \\
\vdots & \vdots & & \vdots \\
0 & 0 & \cdots & 1
\end{array}\right],}
\end{aligned}
$$

or a null block, and

$$
A_{l+m+1,1}=\left[\begin{array}{cccc}
n_{1} & \cdots & n_{y} & n_{y+1} \\
b_{1,1} & \cdots & b_{1, y} & m_{1} \\
\vdots & & \vdots & \vdots \\
b_{y, 1} & \cdots & b_{y, y} & m_{y}
\end{array}\right]
$$

where the submatrix $\left[b_{i, j}\right]$ is defined by the matrix $B_{X}$. The matrices $Z_{k}$ and $N_{j}$ are null matrices, except $N_{l}, N_{l+1}$, and $N_{l+m+2}$ that can contain some elements 1 . The distribution of the previous blocks $A_{i, j}$, with $(i, j) \neq(l+m+1,1)$, is given by the structure of the matrix $A_{V}$. On the other hand, the internal structure of each block $A_{i, j}$ is determined by the order of the shifts of the sequence $W$. For the case of the block $A_{l+m+1,1}$, its submatrix 
$\left[b_{i, j}\right]$ has an internal structure determined by $B_{X}$. The elements $n_{i}$ and $m_{j}$ are null, except those needed to preserve the continuity of the transitions (from the fact that $f$ is a continuous function). Analogously, the block $\bar{A}_{X}$ is determined by $\hat{B}_{X}$. Finally, the blocks $N_{l}, N_{l+1}$, and $N_{l+m+2}$ are null, except, once again, for the elements needed to preserve the continuity of the transitions.

The following example illustrates the use of the previous theorem.

Example 4.2. Let $V=(R M M A, L M M B)$ and $W=(r \ell c, r \ell c)$. Then, we have $S=V * W=$ (RMMMRMMLRMMA,LMMMLMMRLMMB), and the $\otimes$-product of their matrices

$$
A_{V}=\left[\begin{array}{lllllll}
0 & 0 & 0 & 0 & 1 & 1 & 1 \\
0 & 0 & 0 & 0 & 0 & 0 & 1 \\
0 & 0 & 0 & 0 & 0 & 1 & 0 \\
0 & 0 & 1 & 1 & 1 & 0 & 0 \\
0 & 1 & 0 & 0 & 0 & 0 & 0 \\
1 & 0 & 0 & 0 & 0 & 0 & 0 \\
1 & 1 & 1 & 0 & 0 & 0 & 0
\end{array}\right], \quad A_{W}=\left(\left[\begin{array}{ll}
0 & 1 \\
1 & 1
\end{array}\right],\left[\begin{array}{ll}
0 & 1 \\
1 & 1
\end{array}\right]\right)
$$

$A_{S}=A_{V * W}$, is given by

$$
\left[\begin{array}{lllllllllllllllllllllll}
0 & 0 & 0 & 0 & 0 & 0 & 0 & 0 & 0 & 0 & 0 & 0 & 1 & 0 & 0 & 0 & 0 & 0 & 0 & 0 & 0 & 0 & 0 \\
0 & 0 & 0 & 0 & 0 & 0 & 0 & 0 & 0 & 0 & 0 & 0 & 0 & 1 & 0 & 0 & 0 & 0 & 0 & 0 & 0 & 0 & 0 \\
0 & 0 & 0 & 0 & 0 & 0 & 0 & 0 & 0 & 0 & 0 & 0 & 0 & 0 & 1 & 1 & 1 & 1 & 1 & 1 & 1 & 1 & 0 \\
0 & 0 & 0 & 0 & 0 & 0 & 0 & 0 & 0 & 0 & 0 & 0 & 0 & 0 & 0 & 0 & 0 & 0 & 0 & 0 & 0 & 0 & 1 \\
0 & 0 & 0 & 0 & 0 & 0 & 0 & 0 & 0 & 0 & 0 & 0 & 0 & 0 & 0 & 0 & 0 & 0 & 0 & 0 & 0 & 1 & 1 \\
0 & 0 & 0 & 0 & 0 & 0 & 0 & 0 & 0 & 0 & 0 & 0 & 0 & 0 & 0 & 0 & 0 & 0 & 0 & 0 & 1 & 0 & 0 \\
0 & 0 & 0 & 0 & 0 & 0 & 0 & 0 & 0 & 0 & 0 & 0 & 0 & 0 & 0 & 0 & 0 & 0 & 0 & 1 & 0 & 0 & 0 \\
0 & 0 & 0 & 0 & 0 & 0 & 0 & 0 & 0 & 0 & 0 & 0 & 0 & 0 & 0 & 0 & 0 & 0 & 1 & 0 & 0 & 0 & 0 \\
0 & 0 & 0 & 0 & 0 & 0 & 0 & 0 & 0 & 0 & 0 & 0 & 0 & 0 & 0 & 0 & 0 & 1 & 0 & 0 & 0 & 0 & 0 \\
0 & 0 & 0 & 0 & 0 & 0 & 0 & 0 & 0 & 0 & 0 & 0 & 0 & 0 & 0 & 0 & 1 & 0 & 0 & 0 & 0 & 0 & 0 \\
0 & 0 & 0 & 0 & 0 & 0 & 0 & 0 & 0 & 0 & 0 & 0 & 0 & 0 & 0 & 1 & 0 & 0 & 0 & 0 & 0 & 0 & 0 \\
0 & 0 & 0 & 0 & 0 & 0 & 0 & 0 & 1 & 1 & 1 & 1 & 1 & 1 & 1 & 0 & 0 & 0 & 0 & 0 & 0 & 0 & 0 \\
0 & 0 & 0 & 0 & 0 & 0 & 0 & 1 & 0 & 0 & 0 & 0 & 0 & 0 & 0 & 0 & 0 & 0 & 0 & 0 & 0 & 0 & 0 \\
0 & 0 & 0 & 0 & 0 & 0 & 1 & 0 & 0 & 0 & 0 & 0 & 0 & 0 & 0 & 0 & 0 & 0 & 0 & 0 & 0 & 0 & 0 \\
0 & 0 & 0 & 0 & 0 & 1 & 0 & 0 & 0 & 0 & 0 & 0 & 0 & 0 & 0 & 0 & 0 & 0 & 0 & 0 & 0 & 0 & 0 \\
0 & 0 & 0 & 0 & 1 & 0 & 0 & 0 & 0 & 0 & 0 & 0 & 0 & 0 & 0 & 0 & 0 & 0 & 0 & 0 & 0 & 0 & 0 \\
0 & 0 & 0 & 1 & 0 & 0 & 0 & 0 & 0 & 0 & 0 & 0 & 0 & 0 & 0 & 0 & 0 & 0 & 0 & 0 & 0 & 0 & 0 \\
0 & 0 & 1 & 0 & 0 & 0 & 0 & 0 & 0 & 0 & 0 & 0 & 0 & 0 & 0 & 0 & 0 & 0 & 0 & 0 & 0 & 0 & 0 \\
1 & 1 & 0 & 0 & 0 & 0 & 0 & 0 & 0 & 0 & 0 & 0 & 0 & 0 & 0 & 0 & 0 & 0 & 0 & 0 & 0 & 0 & 0 \\
1 & 0 & 0 & 0 & 0 & 0 & 0 & 0 & 0 & 0 & 0 & 0 & 0 & 0 & 0 & 0 & 0 & 0 & 0 & 0 & 0 & 0 & 0 \\
0 & 1 & 1 & 1 & 1 & 1 & 1 & 1 & 1 & 0 & 0 & 0 & 0 & 0 & 0 & 0 & 0 & 0 & 0 & 0 & 0 & 0 & 0 \\
0 & 0 & 0 & 0 & 0 & 0 & 0 & 0 & 0 & 1 & 0 & 0 & 0 & 0 & 0 & 0 & 0 & 0 & 0 & 0 & 0 & 0 & 0 \\
0 & 0 & 0 & 0 & 0 & 0 & 0 & 0 & 0 & 0 & 1 & 0 & 0 & 0 & 0 & 0 & 0 & 0 & 0 & 0 & 0 & 0 & 0
\end{array}\right] .
$$




\section{Acknowledgment}

The authors gratefully acknowledge the financial support from the project FCT-POCTIFEDER.

\section{References}

[1] M. J. Behe, Darwin's Black Box, Touchstone, New York, 1996.

[2] A. Boyarsky and P. Góra, A dynamic system interpretation of irreducible complexity, Discrete Dyn. Nat. Soc. 7 (2002), no. 1, 23-26.

[3] P. Collet and J.-P. Eckmann, Iterated Maps on the Interval as Dynamical Systems, Progress in Physics, vol. 1, Birkhäuser Boston, Massachusetts, 1980.

[4] B. Derrida, A. Gervois, and Y. Pomeau, Iteration of endomorphisms on the real axis and representation of numbers, Ann. Inst. H. Poincaré Sect. A (N.S.) 29 (1978), no. 3, 305-356.

[5] J. P. Lampreia, A. Rica da Silva, and J. Sousa Ramos, $\otimes$-product of Markov matrices, Stochastica 12 (1988), no. 2-3, 149-166.

[6] J. P. Lampreia, R. Severino, and J. Sousa Ramos, Renormalizations for trimodal maps, Differential Equations with Applications to Biology (Halifax, Nova Scotia, 1997), Fields Inst. Commun., vol. 21, American Mathematical Society, Rhode Island, 1999, pp. 353-362.

[7] J. P. Lampreia and J. Sousa Ramos, Symbolic dynamics of bimodal maps, Portugal. Math. 54 (1997), no. 1, 1-18.

[8] J. Llibre and P. Mumbrú, Extending the *-product operator, European Conference on Iteration Theory (Batschuns, 1989), World Scientific Publishing, New Jersey, 1991, pp. 199-214.

[9] R. S. MacKay and C. Tresser, Boundary of topological chaos for bimodal maps of the interval, J. London Math. Soc. (2) 37 (1988), no. 1, 164-181.

[10] J. Milnor and W. Thurston, On iterated maps of the interval, Dynamical Systems (College Park, Md, 1986/87) (J. C. Alexander, ed.), Lecture Notes in Math., vol. 1342, Springer-Verlag, Berlin, 1988, pp. 465-563.

[11] S.-L. Peng and X.-S. Zhang, The generalized Milnor-Thurston conjecture and equal topological entropy class in symbolic dynamics of order topological space of three letters, Comm. Math. Phys. 213 (2000), no. 2, 381-411.

J. P. Lampreia: Departamento de Matemática, Faculdade de Ciências e Tecnologia, Universidade Nova de Lisboa, Lisboa, Portugal

E-mail address: jpl@fct.unl.pt

R. Severino: Departamento de Matemática, Universidade do Minho, 4710-057 Braga, Portugal

E-mail address: ricardo@math.uminho.pt

J. Sousa Ramos: Departamento de Matemática, Instituto Superior Técnico, Universidade Técnica de Lisboa, Lisboa, Portugal

E-mail address: sramos@math.ist.utl.pt 


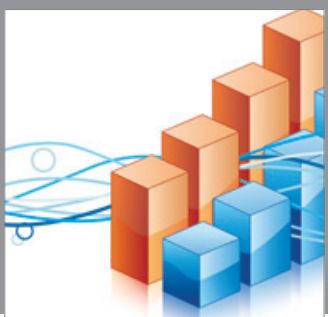

Advances in

Operations Research

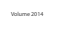

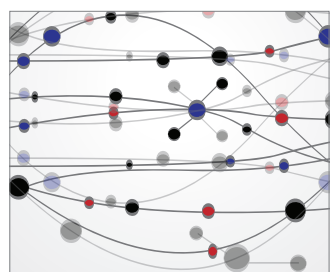

\section{The Scientific} World Journal
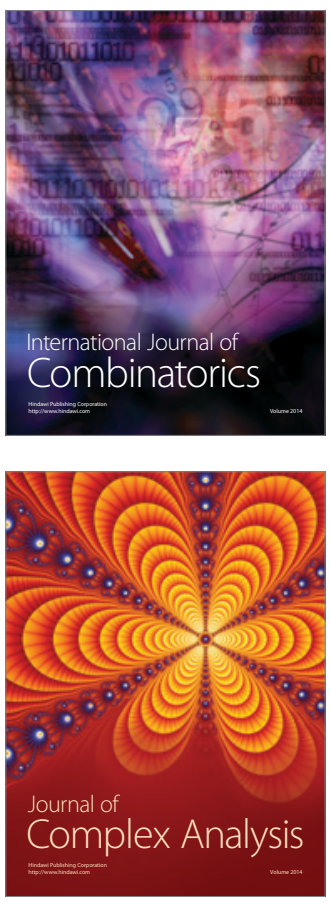

International Journal of

Mathematics and

Mathematical

Sciences
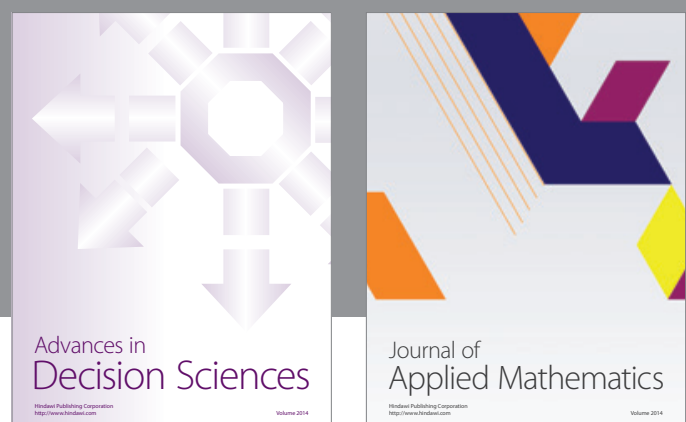

Journal of

Applied Mathematics
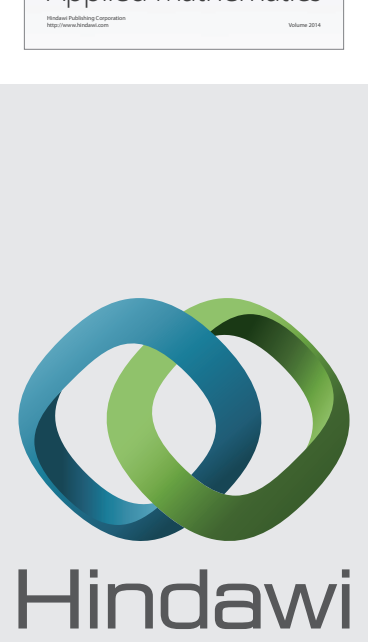

Submit your manuscripts at http://www.hindawi.com
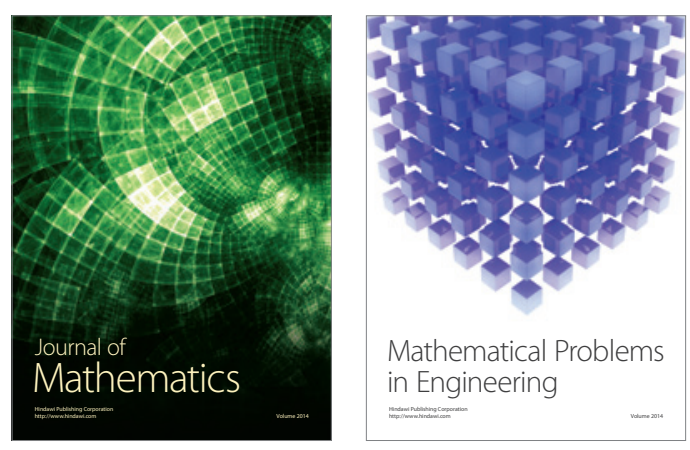

Mathematical Problems in Engineering
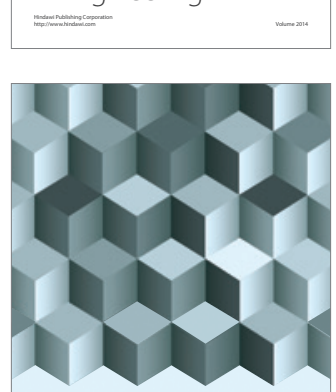

Journal of

Function Spaces
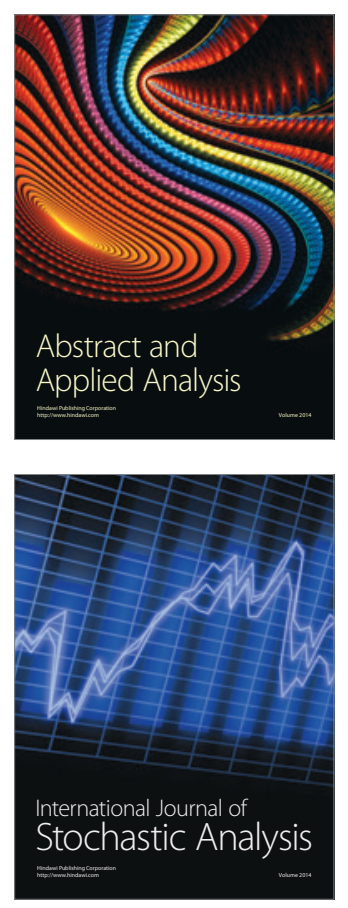

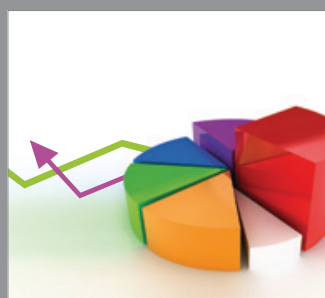

ournal of

Probability and Statistics

Promensencen
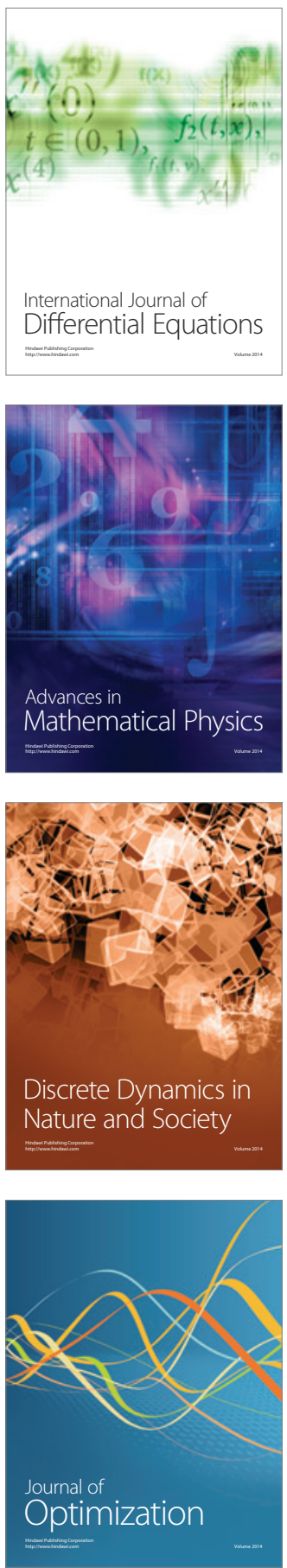